\title{
Simultaneous Bennett's fracture and metacarpophalangeal dislocation of the same thumb in a soccer player
}

\author{
S Jari, M Waseem, M S Srinivasan
}

\begin{abstract}
Double dislocations of finger joints are rare. An unusual case of a simultaneous Bennett's fracture/dislocation of the carpometacarpal joint and a dorsal dislocation of the metacarpophalangeal joint of the same thumb, and the management of this injury are reported. The patient had an excellent functional result.

(Br F Sports Med 2000;34:463-464)
\end{abstract}

Keywords: finger; thumb; Bennett's fracture; dislocation

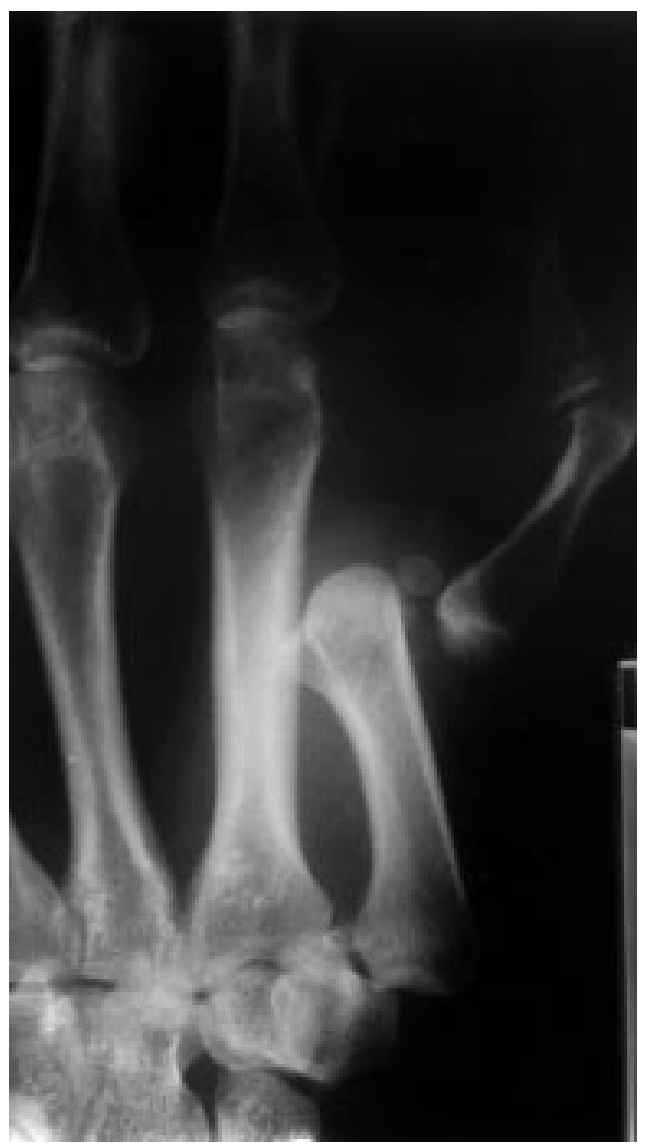

Figure 1 Initial anteroposterior radiograph after the injury showing the simultaneous Bennett's fracture of the first metacarpal base and the dorsal dislocation of the metacarpophalangeal joint of the thumb.
Double dislocations of finger joints are rare. ${ }^{1-6}$ Previous reports of simultaneous double thumb dislocations have included dislocation of the thumb metacarpophalangeal and carpometacarpal joints, ${ }^{36}$ metacarpophalangeal and interphalangeal joints, ${ }^{5}$ and a case of interphalangeal and carpometacarpal dislocation which also included a small avulsion flake of volar beak from the base of the metacarpal. ${ }^{7}$

We report an unusual case of a simultaneous Bennett's fracture/dislocation of the carpometacarpal joint and a dorsal dislocation of the metacarpophalangeal joint of the same thumb.

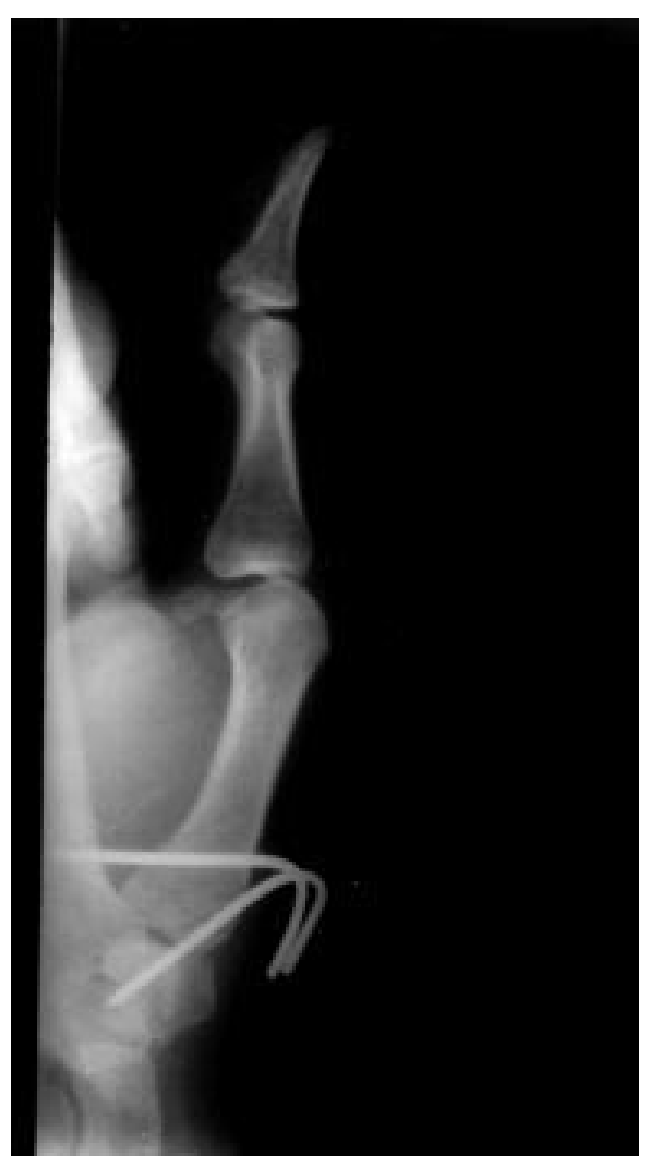

Figure 2 Anteroposterior radiograph after surgery showing reduction and wiring of the first metacarpal base and reduction of the thumb metacarpophalangeal joint. 


\section{Case report}

A 23 year old right handed man fell on to his outstretched left thumb after being tripped during a tackle while playing a game of soccer. He presented to the accident and emergency department with a painful deformed left thumb and no other injuries. He had no neurovascular deficit of the thumb.

A plain radiograph showed a Bennett's fracture/dislocation of the carpometacarpal joint and a dorsal dislocation of the metacarpophalangeal joint of the thumb (fig 1). Under regional anaesthetic block, the dislocations were easily reduced in the accident and emergency department. The metacarpophalangeal joint reduction was stable but the carpometacarpal joint reduction was very unstable. The thumb was therefore temporarily held in a Bennett's type plaster cast and the next morning the patient was taken to the operating theatre. Under general anaesthesia, the stability of the reduced metacarpophalangeal joint was confirmed and the colateral ligaments felt to be intact. The carpometacarpal joint was reduced and the position held using two percutaneous $\mathrm{K}$ wires, one transfixing the carpometacarpal joint and the other through into the base of the second metacarpal (fig 2). A Bennett's plaster cast was reapplied.

The wires were removed after three weeks and the stability of the metacarpophalangeal and carpometacarpal joints reconfirmed. The patient was referred for hand therapy.

Three months after the injury, the patient was reviewed. He had no pain or residual deformity of the thumb. The fracture had united and the carpometacarpal and metacarpophalangeal joints were stable. He had regained a full range of all thumb movements as compared with the other side and had equal thumb-index spans of $17 \mathrm{~cm}$ bilaterally. He had returned to all his normal activities.
He was discharged from further follow up after one year.

\section{Discussion}

The mechanism of injury for carpometacarpal joint dislocation is usually a longitudinally directed force with the metacarpal in slight flexion, whereas the metacarpophalangeal joint injury requires forced hyperextension of the joint. ${ }^{2}$ It is felt that the injuries to the patient's thumb occurred to the distal joint first so as to fulfil both mechanisms.

Once reduced, the metacarpophalangeal joint was stable and the carpometacarpal joint fracture/dislocation could then be treated on its own merit based on the standard treatment protocols for this injury. ${ }^{2}$

We report a rare case of simultaneous Bennett's fracture/dislocation of the carpometacarpal joint and dorsal dislocation of the metacarpophalangeal joint of the thumb and its management. This patient had no limitation of activities after treatment of this injury.

1 Andersen MB, Johannsen H. Double dislocation of the interphalangeal joints in the finger. Scand $\mathcal{F}$ Plast Reconstr Hand Surg 1993;27:233-6.

2 Green DP, Butler TE. Fractures and dislocations in the hand. In: Rockwood CA, Green DP, Bucholz RW, et al, eds. Fractures in adults. 2nd ed. Philadelphia: Lippincott-Raven, Fractures in adults.

3 Ibrahim S, Noor MA. Simultaneous dislocations of the carpometacarpal and metacarpophalangeal joints of the thumb. Injury 1993;24:343-4.

4 Ikpeme JO. Dislocation of both interphalangeal joints of one finger. Injury 1997;9:68-70.

5 Levy IM, Liberty S. Simultaneous dislocation of the interphalangeal and metacarpophalangeal joints of the thumb: a case report. F Hand Surg [Am] 1979;4:489-90.

6 Moore JR, Webb CA, Thompson RC. A complete dislocation of the thumb metacarpal. F Hand Surg [Am] 1978;6:547-9.

7 Wee JTK, Chandra D, Satku K. Simultaneous dislocations of the interphalangeal and carpometacarpal joints of the thumb: a case report. 7 Hand Surg [Br] 1988;13:224-6.

Take home message

Careful assessment of all hand injuries combined with a high index of suspicion will allow diagnosis of uncommon combined injuries such as double fractures/dislocations, permitting correct treatment and leading to an excellent functional recovery. 